Les ANNALES Les Annales de droit

DE DROIT

$11 \mid 2017$

Varia

\title{
La contribution du chercheur en droit à la réflexion sur la transparence
}

The law researcher's contribution to the debate on transparency

Jean-François Kerléo

\section{(2) OpenEdition}

12 Journals

Édition électronique

URL : http://journals.openedition.org/add/536

DOI : $10.4000 /$ add. 536

ISSN : 2606-1988

Éditeur

Presses universitaires de Rouen et du Havre

Édition imprimée

Date de publication : 16 octobre 2017

Pagination : 107-129

ISBN : 979-10-240-0775-5

ISSN : 1955-0855

Référence électronique

Jean-François Kerléo, « La contribution du chercheur en droit à la réflexion sur la transparence », Les Annales de droit [En ligne], 11 | 2017, mis en ligne le 16 octobre 2018, consulté le 19 avril 2019. URL : http://journals.openedition.org/add/536 ; DOI : 10.4000/add.536 


\title{
La contribution du chercheur en droit à la réflexion sur la transparence
}

\author{
Jean-François KeRLÉo
}

Le présent entretien est issu d'un cycle de séminaires consacré aux acteurs de la transparence de la vie publique organisé par l'université de Lille 2 et l'université de Valenciennes sous la direction d'Aymeric Potteau, de Matthieu Caron et d'Annabel Le Moal ${ }^{1}$. Après avoir reçu des élus et des acteurs professionnels, M. Jean-François Kerléo, auteur d'une thèse consacrée à la transparence, a exposé le point de vue du chercheur en droit et l'état de ses réflexions sur le sujet lors d'une conférence qui s'est déroulée le 13 octobre 2016.

Aymeric Potteau. - Notre cycle de séminaires s'intitule «les acteurs de la transparence de la vie publique » mais vous n'avez pas travaillé exclusivement sur la transparence de la vie publique. Comment distinguer précisément le concept de «transparence» et celui de «transparence de la vie publique»?

Jean-François Kerléo. - Ma thèse de doctorat intitulée La transparence en droit. Recherche sur la formation d'une culture juridique avait pour objectif d'étudier l'ensemble des occurrences de la transparence dans le discours du droit et des juristes ${ }^{2}$. Loin d'être limitée à la sphère publique, elle propose une réflexion d'ensemble sur cette notion encore récente - cette terminologie apparaît chez les juristes à la fin des années 1970 - en s'attachant à démontrer, une fois sa généalogie établie, les interactions et les influences qui existent entre ses différents usages. J'ai donc été amené à traiter de matières aussi diverses que le droit des contrats publics, le droit de la santé, le droit de l'environnement, le droit des affaires, le droit pénal, etc. Néanmoins, ce chaos

1. Aymeric Potteau, Matthieu Caron et Annabel Le Moal (dir.), «Cycle de séminaires relatif aux acteurs de la transparence de la vie publique (2015-2017)», Axe droit et finances du CRDP (EA 4487) et IDP de Valenciennes (EA 1384).

2. Jean-François Kerléo, La transparence en droit. Recherche sur la formation d'une culture juridique, Paris, Mare et Martin, janvier 2016. 
apparent du droit peut être, en partie, rationalisé autour de quelques points saillants. L'usage de la transparence, dans les différents régimes juridiques étudiés, révèle toujours une certaine conception de l'État, de la puissance publique, de l'économie et de la concurrence. Autrement dit, la transparence révèle et retranscrit autant qu'elle développe et bouleverse la culture juridique française.

La quasi-totalité des usages de la transparence traduit une interpénétration de la puissance publique et de l'économie. La transparence apparaît ainsi comme le cheval de Troie de l'économie au cœur de l'État. Par les règles de transparence, la puissance publique surveille les relations économiques et organise la concurrence tandis que, dans le même temps, l'économie infiltre la puissance publique pour lui imposer le respect de règles concurrentielles. À mi-chemin entre l'État et le marché, la transparence construit un intérêt général économique qui est indissociable de toute réflexion sur la «transparence de la vie publique». La spécificité de cette dernière résulte, de ce point de vue, de son champ d'application.

C'est dans la culture politique et les conceptions de la représentation et de l'intérêt général, de ses rapports avec l'économie qui traversent les règles juridiques relatives à la transparence de la vie publique que celle-ci trouve son originalité. En effet, cette déclinaison de la transparence n'échappe pas à une certaine approche économique en cherchant - à partir notamment du financement des partis politiques et campagnes électorales, du contenu des déclarations de patrimoine et d'intérêts ${ }^{3}$, des mandats de gestion ou encore, de la réglementation du lobbying - à limiter les connivences entre la vie publique et le monde des affaires. Cette approche diffère d'ailleurs de celle des États-Unis où il s'agit moins de supprimer les liens qui unissent la politique à l'économie, ce qui est caractéristique d'une conception abstraite et idéale de l'intérêt général comme en France (interdiction du financement des partis et des campagnes par les personnes morales depuis 1995 et strict plafonnement pour les personnes physiques ${ }^{4}$ ), que de les rendre publics pour éclairer les citoyens et guider leurs choix électoraux. La transparence «à la française» repose donc sur la pureté de la

3. Ces déclarations peuvent d'ailleurs être entendues comme une nouvelle forme de mise en concurrence politique (inspirée de l'économie) entre les élus, un nouveau mode de sélection des candidats à une élection sur de nouveaux critères renversant la représentation politique par l'idée de "ressembler à ".

4. Voir notamment, L. no 88-227, 11 mars 1988, JO 12 mars 1988, p. 3290 ; L. no 95-65, 19 janv. 1995, JO 21 janv. 1995. 
politique, laquelle doit se détacher des contingences particulières d'ordre économique, financier, etc.

L'association, encore récente, avec la déontologie contribue à une certaine personnalisation de la transparence de la vie publique: les lois du 11 octobre 2013 déshabillent partiellement les élus et leurs collaborateurs en rendant publics leur patrimoine et leurs intérêts ${ }^{5}$. Si cette transparence, qui porte directement sur l'homme, se justifie par le maintien d'une légitimité collective de l'institution à laquelle appartiennent les élus (Assemblée nationale, Sénat, gouvernement, etc.), les lois de 2013, comme celles plus récentes sur la déontologie des fonctionnaires et des magistrats, représentent une brèche dans l'approche française de la transparence. Conformément à une philosophie plus anglo-saxonne que continentale, la mise à nu de la personne privée s'explique par la spécificité de la mission publique dont elle est investie. Outre cette personnalisation qui lui est pour l'instant spécifique (mais qui est étendue, comme au Chili, à certains dirigeants de sociétés privées ou aux propriétaires ou représentants d'organes de presse comme en Turquie), la transparence de la vie publique se dissocie des autres déclinaisons de la notion par un second aspect.

Il existe un conflit d'intérêts inhérent à cette forme de transparence puisque l'État se l'impose à lui-même. Les acteurs publics posent, à travers la transparence de la vie publique, un regard sur eux-mêmes et définissent ce qu'ils veulent refléter vis-à-vis de la société civile. La transparence de la vie publique consiste donc, pour la politique, en une manière de se représenter elle-même, en toute conscience, élaborant ainsi une sorte d'image de l'État et de savoir officiel. Par la transparence, les acteurs publics déterminent également les limites du contrôle susceptible de s'exercer sur eux par les citoyens et la part de liberté nécessaire à leur action.

Hormis ces deux aspects, la transparence de la vie publique est abordable comme n'importe quel usage de la transparence, laquelle se comprend a minima comme un ensemble de techniques comprenant aussi bien des normes juridiques que des représentations idéales fondées sur une réévaluation du rapport savoir/pouvoir en renvoyant à un processus unilatéral ou multilatéral de communication d'informations dans un but de connaissance, d'efficacité ou de légitimation. La spécificité de la transparence de la vie publique ne résulte donc que des interlocuteurs qui sont

5. L. $\mathrm{n}^{\mathrm{o}} 2013-907,11$ oct. 2013 relative à la transparence de la vie publique, JO 12 oct. 2013 , p. 16829 ; L. organique $\mathrm{n}^{\circ} 2013-906,11$ oct. 2013 relative à la transparence de la vie publique, JO 12 oct. 2013, p. 16824. 
concernés et du rapport particulier qui existe entre eux. Autrement dit, cette transparence met en place un rapport de force entre l'État et les citoyens à partir de nouvelles formes d'organisation, de contrôle et de légitimation politiques.

Annabel Le Moal. - Comment doit se positionner le chercheur par rapport à la question de la transparence? Est-il là pour simplement révéler ou proposer? Pour dire «ce qui est» ou pour dire " ce qui doit être » ? En somme, quelle est la méthode que le chercheur doit retenir pour travailler sur cette question?

Jean-François Kerléo. - Je ne dirais pas que la réponse à ces questions se pose en termes de devoir. La méthode est un choix personnel fondé sur son propre système de valeurs. L'alternative entre le positivisme et le jusnaturalisme repose sur une certaine interprétation du monde. Méthode et fond sont consubstantiellement liés puisque l'approche que vous retenez détermine le sens de votre analyse. Plus précisément encore, la méthode construit son objet d'étude. Si vous choisissez une posture positiviste, vous n'apprécierez pas la transparence en termes de valeur, bien ou mal, juste ou injuste, mais vous considérerez qu'elle entre, par exemple, en contradiction avec la définition de la vie privée du juge constitutionnel (qui a d'ailleurs censuré l'obligation de mentionner les activités des parents et enfants des élus dans les déclarations d'intérêts) ou que les définitions d'accès, de diffusion, de réutilisation d'un côté, d'actes administratifs, de documents administratifs, de données personnelles ou encore d'informations publiques, d'un autre côté, ne se recoupent pas d'une loi à l'autre.

De nombreux choix s'offrent au chercheur qui souhaite étudier une notion. Il peut, par exemple, définir lui-même la transparence afin d'étudier les règles juridiques qui correspondent à son approche stipulative. Cette approche m'est apparue inadaptée à l'étude de la transparence, dans la mesure où je souhaitais étudier la totalité des occurrences du terme dans le discours juridique et, justement, comprendre pourquoi ce terme est parfois utilisé et d'autre fois rejeté. Une conception plus analytique, que j'ai retenue, exige de prendre le langage pour objet d'analyse afin d'étudier certaines règles juridiques. Même dans cette hypothèse, une prédéfinition de l'objet et du champ d'analyse est nécessaire. $\mathrm{Si}$, intuitivement, la transparence parle à tout le monde, elle apparaît plus complexe lorsque l'on cherche à la définir précisément. Elle est un donné du discours qui reste à construire à partir d'une méthodologie originale. Plutôt que de dresser un catalogue des innombrables manifestations de la 
transparence, j'ai élaboré une méthode permettant d'appréhender la notion dans sa diversité. J'ai considéré qu'il n'y avait « transparence » qu'en présence du terme dans le discours. J'ai donc cherché à comprendre pourquoi, à propos d'un même régime juridique (accès aux documents administratifs, motivation des actes individuels défavorables, etc.), la transparence était ou non utilisée. Autrement dit, quelles représentations elle recouvrait et quelles utilités elle avait dans et pour le discours du droit. Il ne s'agissait donc pas, ce qui est parfois déroutant pour un juriste, de commenter un régime juridique déterminé. De même, je n'ai pas cherché à aboutir à une stricte définition de la transparence en droit mais, plutôt, à saisir les articulations entre les usages de la transparence afin de révéler les structures intellectuelles des juristes (producteurs de droit et doctrine) et leur influence sur celles-ci.

J'ai tenté de pénétrer, à travers la notion de transparence, l'univers mental des juristes. J'ai alors enrichi ma thèse de réflexions philosophiques sur l'imaginaire, d'auteurs tels que Cornélius Castoriadis, Michel Foucault et Paul Veyne. Cette approche concorde avec le courant américain des "Cultural Studies of Law», et notamment les travaux de Paul Kahn qui considère le droit comme un phénomène culturel agissant sur nos consciences, nos croyances et nos représentations ${ }^{6}$. Le choix de cette méthode originale a permis d'apporter un éclairage nouveau sur la transparence. J'ai mis à jour la structure de l'imaginaire juridique de ceux qui utilisent ou, au contraire, rejettent la transparence, ainsi que les raisons qui les poussent à s'engager dans un processus constant de réification de la notion. J'ai cherché à répondre à différentes questions qui me semblaient essentielles. Comment les juristes imaginent-ils le droit quand ils invoquent la transparence ? Comment la transparence se dote-t-elle de sens à partir de cet imaginaire des juristes? Comment ce mot en vient à sembler si "naturel" et à structurer à son tour les contours de la pensée juridique dans les branches du droit les plus variées (financement des partis politiques et campagnes électorales, transparence fiscale ou tarifaire, notification des aides d'État, passation des marchés publics, tarification de l'accès aux infrastructures essentielles, procès équitable, opinions dissidentes, etc.) ? Quels liens invisibles relient tous ces objets juridiques?

6. Paul W. Kahn, The Cultural Study of Law. Reconstructing Legal Scholarship, University of Chicago Press, 1999. Voir également David Nelken, « Defining and Using the Concept of Legal Culture», dans Comparative Law : A Handbook, Esin Örücü et David Nelken (dir.), Oxford, Hart, 2007, p. 109-132; David Nelken, «Using the Concept of Legal Culture ", Australian Journal of Legal Philosophy, $\mathrm{n}^{\circ}$ 1, 2004, p. 1-28. 
Matthieu Caron. - Quelle est votre philosophie de la transparence? Doit-on tout dire, tout dévoiler?

Jean-François Kerléo. - Cette question exige de distinguer ma «philosophie personnelle», ce que je considère comme bien et mal, de ma "philosophie scientifique» qui correspond à ma méthode d'analyse de l'objet transparence et que j'ai évoquée préalablement. La première est parfois déterminée par la seconde, si l'on rejette notamment la neutralité axiologique du positivisme pour une approche critique du droit ${ }^{7}$. De cette manière, les opinions personnelles sont incluses dans la méthodologie dont l'approche consiste, en se fondant sur des conceptions idéologiques (politiques, sociales, économiques, etc.), à porter un jugement de valeur sur son objet d'étude. Cette approche n'est pas la mienne puisque, au contraire, je cherche dans le droit à saisir les idéologies qui le (sur)déterminent pour en comprendre l'ontologie (entendue de manière relative et non essentielle).

Ce qui est ici visé dans les questions posées concerne donc la sphère des valeurs, c'est-à-dire ma position critique vis-à-vis des règles juridiques elles-mêmes. Je ne peux donc y répondre qu'en tant que citoyen, et non comme scientifique. Certes, mon opinion est influencée par mes propres travaux qui m'amènent à m'interroger continuellement d'une manière originale et approfondie sur cette question, cloisonner l'opinion du citoyen de l'éclairage du juriste serait évidemment absurde. Je suis, pour paraphraser Georges Burdeau, un "homme situé» qui s'exprime en fonction de ses compétences et de ses expériences ${ }^{8}$. Dans cette optique, toute opinion sur la transparence est, selon moi, indissociable d'une appréciation sur la culture dans laquelle celle-ci s'inscrit, et notamment sur le rôle de l'État, de l'opinion publique et de la diffusion du savoir.

Dans une société de l'information où les nouvelles technologies donnent un accès quasi illimité à l'information, le droit doit organiser la diffusion des données afin d'éviter que ne s'installe un climat de défiance qui est provoqué par la publication de révélations (du moins présentées comme telles) en dehors de toute obligation juridique ${ }^{9}$. Le droit pose les limites de l'utile, du convenable et de l'acceptable. Un doute

7. Sur ces questions voir Max Weber, Le savant et le politique, Paris, Union générale d'éditions, 1963.

8. Georges Burdeau, La démocratie. Essai synthétique, Bruxelles, Office de Publicité, 1956.

9. Yves Jégouzo, «La modernisation et la transparence de l'administration au défi de Wikileaks», AJDA 2010, p. 2449. 
subsiste toujours là où il n'intervient pas en raison de la correspondance qui est trop souvent établie entre le secret et la volonté de cacher. Quand, tout d'un coup, surgissent dans la presse des informations sur les liens ou les intérêts d'un élu, une réaction immédiate consiste à croire qu'il est un intrigant, de mauvaise foi, qu'il agit pour son intérêt personnel. Les tensions sont alors désamorcées par l'établissement préalable de règles du jeu, lesquelles doivent régir les comportements individuels. Lorsque j'évoque l'intervention du droit, ce n'est pas pour qu'il impose systématiquement la transparence mais, plutôt pour qu'il en définisse les contours afin de déterminer les poches intangibles du secret (l'infranchissable), et d'en expliquer le bien-fondé. Par exemple, dans un tout autre registre, l'exploitation de la faiblesse de certains publics, dont les jeunes, par des personnes mal intentionnées qui les incitent à des pratiques d'exhibition sur Internet qu'ils diffusent ensuite sur les réseaux sociaux, doit être prise en charge par le droit pour prévenir les dangers de la «transparence du corps ${ }^{10} »$.

Quant aux limites à la transparence de la vie publique qu'évoque la question ici posée, aucune réponse type ne peut être apportée. La transparence s'inscrit dans une globalité que l'on peut encore apprécier du point de vue étatique, en prenant évidemment en considération les influences extérieures (droit international, Union européenne, pression d'associations ou d'organismes de lutte contre la corruption, etc.). Chaque déclinaison de la transparence se comprend à l'intérieur d'une culture particulière qui accepte par exemple la mise à nu d'un élu en fonction de la place accordée à l'opinion publique, aux médias, à la liberté individuelle (vie privée), à la liberté d'expression, au droit de propriété, etc. Aux États-Unis, la totalité des déclarations de patrimoine est accessible pendant 6 ans, à la seule exception de celles des fonctionnaires des douanes et des impôts, des agents de passation des marchés et des autorités financières. Au contraire, s'il existe une déclaration d'intérêts, la Suisse n'en prévoit pas pour le patrimoine, le droit de propriété restant sacré, et la Belgique comme le Japon refusent de la rendre publique. Porter un jugement sur la transparence exige toujours d'apprécier le bouleversement qu'elle provoque dans les institutions au sein d'un système donné de représentations culturelles.

10. Voir par exemple l'article 227-22-1 du Code pénal (L. n ${ }^{0}$ 2007-297, 5 mars 2007 [art. 35], JO 7 mars 2007). À l'inverse, la «transparence des corps» est parfois imposée dans l'espace public (L. ${ }^{\circ}$ 2010-1192, 11 oct. 2010 interdisant la dissimulation du visage dans l'espace public, JO 12 oct. 2010, p. 18344). 
En France, la transparence progresse par petites touches en fonction, non des besoins, mais des scandales. La loi sur la protection des données personnelles qui créa en janvier 1978 la CNIL intervient à la suite du "scandale Safari»; la loi du 11 mars 1988 sur la transparence de la vie publique est consécutive à «l'affaire Luchaire» et, plus récemment, les lois du 11 octobre 2013 ont été adoptées à la suite de «l'affaire Cahuzac» : autant dire que la transparence n'appartient que marginalement à la culture française des élites. À titre personnel, je regrette que la transparence n'évolue qu'en fonction des scandales et que, d'une manière générale, le droit français ne s'adapte aux mouvements de la société qu'en cas de crise ou de contestation sociale. En revanche, la transparence ne doit pas bouleverser les cadres culturels de l'État mais, plutôt, les faire progressivement évoluer. Il convient de ne pas forcer trop brutalement certains verrous, en publiant, par exemple, les déclarations patrimoniales des magistrats, ce qui bouleverserait les cadres théoriques de la justice et du jugement par la remise en cause de la conception syllogistique (c'est-à-dire mécanique) de la décision juridictionnelle, et en instillant un doute malvenu sur la neutralité, l'impartialité, et même le désintéressement du juge au cours d'un procès.

Les réticences vis-à-vis de la transparence s'expliquent également par une conception restreinte de la liberté d'expression : la place de l'opinion publique reste modeste et les opinions exprimées assez formalistes, contrairement à d'autres États où la sacralisation de l'action publique n'est pas moins importante. Ceci explique que le développement de la transparence ne soit pas vraiment réfléchi en termes démocratiques, à partir d'une volonté d'éclairer le citoyen et de développer la connaissance. La sphère publique, entendue comme le lieu de discussion entre les différentes forces vives de la société, est assez peu développée en France, et les enjeux électoralistes cristallisent finalement les débats. La conception de la représentation politique, assez sclérosante pour l'opinion publique, nourrit la controverse dans les seuls moments électoraux. Dès lors, le développement de la transparence, et notamment la politique active d'open data, est porté presque exclusivement par des considérations mercantiles et budgétaires, les données publiques constituant de véritables gisements économiques permettant l'ouverture de nouveaux marchés et réduisant les coûts de fonctionnement de l'administration ${ }^{11}$.

11. L'étude d'impact de la loi sur la République numérique est assez éloquente, elle va même jusqu'à évoquer la réduction des coûts de transaction (L. $\mathrm{n}^{\circ} 2016-1321,7$ oct. 2016 pour une République numérique, JO 8 oct. 2016). Si l'économie constitue une 
À quand une transparence tournée vers l'appropriation libre et éclairée de l'État par les citoyens?

Annabel Le Moal. - Qu'est-ce qui distingue le travail du chercheur et du journaliste en matière de transparence de la vie publique? Peuvent-ils se compléter? Sont-ils concurrents?

JeAn-François KerLÉo. - L'objet scruté par le juriste et le journaliste ainsi que les outils utilisés aux fins de l'analyse ne sont pas comparables, ce qui suppose une importante divergence dans leur travail. Le juriste s'intéresse à la norme juridique et éventuellement, selon les approches retenues, à son application par le juge, mais rarement par ses destinataires. Une vision, parfois étriquée, du positivisme juridique exclut du champ d'analyse la pratique du droit, ne permettant pas au chercheur d'apprécier le décalage entre les règles et leur application au sens large (utilité, instrumentalisation, usage, etc.) Plus exactement, l'application des règles de droit est principalement appréciée sous l'angle du contentieux, excluant souvent l'analyse des pratiques réelles qui ne vont pas jusqu'au prétoire. Dans cette optique, le juriste perd une partie de sa place dans la cité puisqu'il s'adresse moins aux citoyens qu'aux professionnels du droit (juges, avocats, agents publics, etc.). Le journaliste prend alors le relais du chercheur en relatant plus franchement la vie concrète du droit.

D'un point de vue schématique, on peut considérer, à propos des déclarations de patrimoine et d'intérêts notamment, que le juriste s'intéresse au contenant, à l'instrumentum, tandis que le journaliste travaille sur le contenu, le negotium. Tandis que le premier va travailler sur le régime des déclarations, notamment sur les difficultés de leur insertion dans l'ordre juridique, et par exemple leur conformité aux libertés fondamentales (liberté d'expression et d'opinion, vie privée, neutralité des agents publics, indépendance des magistrats, etc.), le journaliste va s'emparer des informations qu'elles contiennent pour contrôler la probité des élus et expliquer à partir des intérêts détenus certains comportements, prises de position, etc. Leurs travaux se situent à des niveaux différents, ce qui ne veut pas dire qu'ils ne se recoupent pas.

Les analyses du juriste sur les insuffisances ou les dangers de certaines pratiques, ainsi que sur l'établissement de comparaisons avec les droits

part évidente de la transparence, sa dimension démocratique reste excessivement faible en France. 
étrangers, constituent une source d'informations sur laquelle le journaliste appuie son analyse en vue d'informer le public ${ }^{12}$. Les thèmes abordés et les conclusions qui en sont tirées sont parfois identiques, c'est alors le cheminement intellectuel (c'est-à-dire l'approche) et la technicité qui varieront entre les deux analyses. Mais, de manière générale, le matériau utilisé par le juriste reste dépersonnalisé, son travail ne consistant pas à viser des comportements particuliers ou des personnes nommément désignées. Contrairement au journaliste, une certaine abstraction est de rigueur dans le travail du juriste, qui aborde son objet sous un angle général et impersonnel.

Toujours à propos des déclarations de patrimoine et d'intérêts, la comparaison entre le juriste et le sociologue (voire l'anthropologue ou plus spécifiquement l'ethnologue) et l'économiste s'avère plus pertinente. Contrairement à ces différents chercheurs en sciences humaines, le juriste est assez démuni pour éplucher les déclarations, les comparer, établir des statistiques et en tirer des conclusions quant aux profils des élus et de la classe dirigeante en général. Les implications de la transparence représentent finalement un champ d'activité limité pour le juriste, lequel est mobilisé pour se prononcer sur les enjeux et les dangers (potentiels ou avérés) d'un régime juridique pour la protection des droits de l'homme, la sécurité publique, la lutte contre la corruption... Mais peut-il à proprement parler mesurer l'impact de la transparence sur tous ces points à partir du droit? Rien n'est moins sûr. Le sociologue est mieux armé pour apprécier les effets du droit sur le terrain. L'économiste est également intéressé par la transparence dès lors que les données publiques réduisent les coûts de transaction, selon le concept développé par l'économiste de Chicago Ronald Coase ${ }^{13}$ et deviennent des valeurs échangeables sur le marché. Tout ceci explique, en partie, le désintérêt des juristes pour cette transparence de la vie publique sur laquelle ils n'ont qu'une prise limitée.

Matthieu Caron. - Comment le chercheur que vous êtes conçoit-il le rôle respectif des pouvoirs publics (élus, administration et AAI), des

12. Le journaliste est, selon la $\mathrm{CEDH}$, le chien de garde de la démocratie, rôle que ne remplit pas le chercheur dont les travaux peuvent, en revanche, servir de support à la presse de qualité. Voir notamment CEDH, 15 déc. 2009, Financial Times LTD et autres c/Royaume-Uni, $\mathrm{n}^{\circ}$ 821/03, Actualités droits-libertés du 15 décembre 2009; CEDH, Grande Ch., 27 mars 1996, Goodwin c/Royaume-Uni de Grande-Bretagne, $\mathrm{n}^{\mathrm{o}} 17488 / 90$.

13. Ronald Coase, L'entreprise, le marché et le droit, Boualem Aliouat (trad.), Paris, Éditions d'Organisation, 2005. 
journalistes, des juges et des citoyens (associations, citoyen lambda, lanceurs d'alerte) en matière de transparence?

Jean-François Kerléo. - Votre question est vaste puisqu'elle renvoie à la manière dont s'articulent les différents acteurs de la transparence au sein de l'État. Comme je l'ai déjà abordé sous l'angle des journalistes et des autres disciplines universitaires, je me focaliserai donc sur certains d'entre eux. Il n'y a pas de strictes distinctions entre tous ces acteurs qui peuvent parfois jouer des rôles similaires. La transparence, et c'est ce qui explique le désintérêt relatif de la doctrine juridique pour cet objet, constitue un enjeu pour de nombreuses personnes ou autorités qui se l'approprient, soit parce qu'elles sont chargées d'en contrôler le respect, soit parce qu'elles cherchent à en accroître la portée sur la vie publique.

J'exclus immédiatement l'assimilation du chercheur au lanceur d'alerte. Si les travaux universitaires peuvent conduire à certaines mises en garde sur les dérives de la transparence, il ne s'agit que de dénoncer l'orientation du droit ou le contenu d'un régime juridique. Quant à lui, le lanceur d'alerte dénonce des agissements illégaux, qui touchent des personnes nommément désignées, et dont la réalisation a été rendue possible par l'opacité régnante. Ces deux activités contribuent à interpeller les pouvoirs publics qui sont parfois conduits à améliorer le contrôle, et donc la transparence, dans les domaines d'activité concernés (le droit constitutionnel après l'affaire du sang contaminé, le droit de la santé après l'affaire du Médiator, du Pradaxa, du Gardasil, le droit des données après le scandale du STIC...) Toutefois, ce sont les dénonciations brutales des lanceurs d'alerte qui connaissent une réelle répercussion sur le droit ; l'influence des universitaires sur la vie publique est devenue assez faible en raison du monopole que détient la haute fonction publique sur les réformes législatives et réglementaires. Ce constat me permet d'aborder le second point, qui concerne le pouvoir administratif au sein de l'État français.

La culture nationale est construite autour d'un État administratif, globalement organisé par une administration qui concentre l'expertise technique et se présente comme la garante de la probité des élus (HATVP, CNCCFP, défenseur des droits, etc.). L'administration accroît son dédoublement fonctionnel, puisqu'elle produit à la fois du droit et de la doctrine par ses rapports officiels, les avis, commentaires et articles rédigés par certains hauts fonctionnaires. Dans cette structuration du pouvoir, la place accordée par les pouvoirs publics aux chercheurs est limitée. Les travaux de l'administration et d'une doctrine organique 
de plus en plus présente alimentent évidemment les recherches universitaires mais maintiennent le chercheur dans une dépendance intellectuelle. Il convient de nouer des contacts avec l'administration, qui produit des ressources substantielles (actes, décisions, rapports, etc.) en vue d'obtenir de quoi nourrir sa réflexion, et certaines autorités telles que la HATVP sont d'ailleurs très ouvertes à la collaboration.

On peut toutefois regretter que les élus s'appuient presque exclusivement sur l'expertise administrative et que d'autres voix, parfois plus innovantes, soient moins écoutées. Le parlement, qui procède à de nombreuses auditions pour chaque projet ou proposition de loi, est plus libre vis-à-vis de l'administration que les membres du gouvernement qui consultent les hauts fonctionnaires qu'ils dirigent. En réalité, aucune concurrence ne s'installe entre la haute administration et l'université dans la mesure où cette derrière, à quelques exceptions notables, est maintenue, voire reste de son propre fait, en retrait de la production du droit. Certes, l'activité scientifique exige une réserve, voire une distance, vis-à-vis du pouvoir. Cependant, cette neutralité ne doit pas empêcher l'universitaire de s'exprimer en tant qu'intellectuel dans la cité ni d'ailleurs les élus de récupérer ses travaux pour nourrir les réformes en cours. Le nombre des universitaires auditionnés à l'occasion de la réforme du statut des fonctionnaires, impliquant de nouvelles perspectives pour la transparence, laisse assez perplexe...

Il conviendrait peut-être de développer la présence de personnalités extérieures, notamment des universitaires, dans les autorités administratives où des questions de transparence sont en jeu. Des propositions en ce sens, non adoptées, avaient été faites au parlement lors du vote de la loi créant la HATVP, à l'instar d'autorités comme la CADA, la CNIL, le CSA, le CSM et évidemment le déontologue de l'Assemblée nationale. L'apport serait évident pour tout le monde : regard extérieur, réflexions substantielles et échanges avec les autres spécialistes permettent d'ouvrir de nouvelles perspectives et d'imposer un contrôle moins formaliste.

Enfin, les associations liées à la thématique de la transparence sont nombreuses: Transparency International France, Anticor, Regards citoyens contribuent par leurs travaux, d'une très grande qualité, à une surveillance de la vie publique qui accroît toujours la transparence et crée le débat. Ce tribunal de l'opinion publique, dont l'universitaire pourrait faire partie avec ses expertises indépendantes, joue un rôle indispensable dans un système démocratique. On peut regretter, ici aussi, qu'une cloison étanche existe parfois entre ces associations et les universitaires. Participer aux travaux de ces associations n'est pas forcément synonyme, contrairement à l'opinion répandue, d'engagement idéologique, l'apport 
du point de vue universitaire n'implique pas qu'il adhère à leurs activités politiques de lobbying. Le rapprochement avec ces organismes est d'ailleurs particulièrement intéressant pour saisir le processus de fabrication de la loi, tout particulièrement celle sur la transparence, et comprendre le fonctionnement des pouvoirs publics.

Aymeric Potteau. - Traiter de la transparence, c'est souvent avoir affaire à la question du secret et de l'opacité, comment surmonter cette difficulté méthodologique?

Jean-François Kerléo. - Traiter de la transparence, c'est évidemment s'attaquer à ses obstacles qui sont d'ailleurs plus nombreux que le seul secret puisque s'y opposent également l'inintelligibilité, la complexité, la confidentialité, l'interdiction de réutilisation de données, le conflit d'intérêts, le favoritisme, etc. Toute analyse s'apprécie dans la dialectique, toujours renouvelée, de la transparence et du secret et l'intitulé de l'ouvrage de Jean Starobinski sur Rousseau - La transparence et l'obstacle - l'évoque très bien ${ }^{14}$. La transparence prend sens à partir de ce à quoi elle résiste; elle ne s'impose pas dans un lieu vide mais prend la place déjà occupée par le secret. Par ailleurs, toute tentative de transparence, aussi pure soit-elle, rencontre toujours la résistance du for intérieur, inhérente à la condition humaine.

Le droit fait émerger aussi bien la transparence que le secret: en reconnaissant le secret, le droit délimite la transparence, et inversement. Toutefois, la dialectique est, en droit, assez spécifique puisque la transparence ne s'applique que si elle est explicitement prévue par les textes tandis que le secret n'exige pas de reconnaissance préalable pour s'imposer. En somme, le secret est le principe (implicite), la transparence l'exception. Toutefois, l'évolution du droit, et plus globalement de la société, contribue à faire de la transparence une idéologie en l'attachant à tout objet du monde présent. Quelle réforme, aujourd'hui, ne contient pas une référence à la transparence? Quel que soit le domaine concerné, et même dans les plus étrangers au premier abord à la transparence, celle-ci est mobilisée d'une manière ou d'une autre pour accroître l'information, le contrôle, l'efficacité de quelque chose. En ce sens, elle se présente comme un nouveau paradigme de l'action publique.

Pour comprendre la transparence, il convient donc de saisir ce à quoi elle s'oppose. Que cherche-t-on à remettre en cause dans le

14. Jean Starobinski, Jean-Jacques Rousseau: la transparence et l'obstacle, Paris, Gallimard, 2006. 
préexistant lorsqu'on l'impose à tel ou tel domaine? Or, d'une part, il y a loin, parfois, entre l'effet escompté et les conséquences pratiques, cet écart contribuant à modifier constamment le rapport de force entre la transparence et le secret. Les justifications politiques doivent donc toujours être questionnées. La relation d'opposition, d'autre part, entre ces deux notions n'est pas toujours évidente dans la mesure où elles servent, selon les époques, à atteindre le même but. On revient parfois sur la transparence au nom de ce pour quoi elle s'appliquait, comme en matière de transparence tarifaire, qui était imposée dans les années 1980 avant d'être rejetée pour les mêmes raisons de loyauté économique et de concurrence dans les années $2000^{15}$. De même, la publicité légale des entreprises permet un meilleur contrôle des pouvoirs publics mais, dans le même temps, met à disposition des concurrents des informations qu'ils utilisent pour évincer certains opérateurs économiques du marché et rétablir un oligopole.

Enfin, il est remarquable de constater que la transparence produit de nouvelles formes d'opacité. Il est d'autant plus aisé de se dissimuler qu'il y a beaucoup d'informations disponibles : si la lumière éclaire, elle peut aussi éblouir. La production excessive d'informations produit de l'irrationalité, car nous n'avons pas toujours les moyens de l'exploiter et d'en contrôler la fiabilité : la densité des documents exigés et la multitude des interprétations qui en ressortent produisent une incertitude très forte. On cherche à établir une hypothétique confiance des citoyens envers leurs élus en imposant des déclarations de patrimoine et d'intérêts qui enrichissent la contestation et favorisent la défiance. Le secret est donc toujours un horizon de la transparence aussi bien parce qu'elle s'y oppose que parce qu'elle le complète ou l'enrichit.

Matthieu CARon. - Comment êtes-vous parvenu à accéder à certaines sources, opaques ou secrètes?

Jean-François Kerléo. - Comme je l'exposais précédemment, j’ai travaillé sur les textes juridiques et non directement, à l'instar d'un journaliste ou d'un sociologue, sur le contenu des déclarations de patrimoine et d'intérêts ou autres informations plus factuelles. Si de nombreuses

15. Pour une approche critique de la transparence tarifaire voir Daniel Mainguy, Jean-Louis Respaud et Malo Depincé, Droit de la concurrence, Paris, LexisNexisLitec, 2010, p. 131-162; Louis Vogel et Joseph Vogel, «Ombres et lumières de la transparence tarifaire (conditions générales de vente et facturation) », JCP E 1995, $\mathrm{n}^{\mathrm{o}} 5$, p. 69. 
informations restent opaques en raison de l'absence d'obligation juridique de les rendre accessibles, je me suis contenté, sans chercher à y accéder, de constater l'insuffisance de la transparence dans certains domaines. Dans la mesure où le droit n'impose pas la diffusion ou l'accès, de nombreuses informations secrètes n'en sont finalement pas, c'est-à-dire qu'elles n'existent pas à proprement parler. Cette fabrication de l'information dépend exclusivement du droit qui, en en exigeant la communication, contribue à les créer ${ }^{16}$. Traiter de la transparence, c'est donc travailler sur la manière dont l'information est fabriquée par les institutions ou les groupes socio-économiques, est engendrée puis communiquée dans l'espace public. Il convient, dès lors, de travailler sur les structures de l'État, lesquelles ne s'appréhendent pas aisément avec le regard externe du chercheur tant les logiques internes et les rapports de force intérieurs comme extérieurs sont déterminants pour saisir la transparence publique.

La difficulté de mon travail, surtout pour la thèse, a été de réunir une somme de textes juridiques et de les analyser en vue d'effectuer des comparaisons entre les différents régimes relatifs à la transparence. C'est le nombre de sources, dans des disciplines très variées du droit, et parfois leur ancienneté, qui ont été les principaux obstacles à mon travail de chercheur. Ne pouvant prétendre être spécialiste de tous les domaines dans lesquels la transparence est employée, il a fallu composer avec la technicité de certaines matières pour parvenir à développer une pensée plus universaliste. Je n'ai donc pas été confronté au secret ou à l'opacité en ce sens où l'accès à ces textes n'a pas été compliqué mais, au contraire, d'autant plus facile que les textes sur la transparence servent très souvent d'affichage politique. Tout nouveau régime de transparence, qui constitue un véritable processus de légitimation, s'accompagne en général d'une large publicité qui s'explique par l'association qui est opérée dans l'esprit des élus comme des citoyens entre transparence et probité. Adopter un texte sur la transparence revient, d'une manière assez magique, à affirmer son intégrité. Sur ce point, un travail sur le titre des textes juridiques serait particulièrement fécond, la transparence étant mentionnée au frontispice d'une règle de droit en contradiction avec son contenu.

16. Il n'y a pas d'informations sur la provenance réelle d'un amendement (qui peut être rédigé clé en main par un lobby, un professionnel, le député lui-même, etc.) tant que le droit ne l'exige pas: il est strictement impossible d'en connaître l'origine, si ce n'est de fouiller dans la messagerie électronique des ministres ou parlementaires, ce qui n'est pas conseillé, même au nom de la qualité du travail universitaire! 
Une autre difficulté est apparue pour l'historicité de la transparence. C'est ici que le travail sur les sources a été parfois compliqué puisqu'il a fallu rechercher des textes relatifs à la publication et à l'accès aux actes juridiques ou à des débats et, plus généralement, aux modalités retenues dans le droit ancien pour porter les règles à la connaissance de ses destinataires ${ }^{17}$. Ce travail a été avant tout celui d'un historien du droit avec, toutefois, la particularité qu'il ne s'agissait pas d'étudier précisément le droit d'une société disparue mais d'établir une corrélation entre des règles juridiques, dans le but de saisir comment et pourquoi la transparence contient, dans son être même, certaines représentations qui (sur)déterminent ses usages d'aujourd'hui. Dans cette optique, j'ai opté pour une approche archéologique que Paul Veyne définit comme une "philosophie de la relation ${ }^{18}$ ", laquelle prémunit contre l'illusion d'une genèse, d'une origine ou d'une source première. Je n'ai donc pas cherché à établir une causalité historique à partir de "notionsancêtres" à la transparence ni à déterminer les raisons intangibles de son apparition. Il s'est agi de repérer des filiations conceptuelles, des lignes de force dans le discours à travers les continuités et les discontinuités dans la pensée juridique et ses représentations. Cette orientation, très féconde, n'est pas sans difficulté puisqu'il s'agit de déchiffrer les structures de pensée qui ont contribué à l'émergence puis à l'évolution de l'idéologie de la transparence. De la légitimation par la publicité parlementaire d'un nouvel ordre politique à la Révolution en passant par la justification de l'administrativisation du pouvoir politique, de l'étatisation du financement des partis et de l'économisation de la puissance publique, la transparence s'est intégrée à de multiples schémas idéologiques qu'une approche kaléidoscopique a permis de saisir dans sa généralité sans rogner sur l'analyse technique du droit.

Matthieu Caron. - Quels sont les domaines dans lesquels demeurent secret et opacité?

17. J'ai effectué un rapprochement consubstantiel entre l'instrumentalisation de la publicité parlementaire développée à partir de la Révolution française et l'usage actuel de la transparence.

18. Paul Veyne, Comment on écrit l'histoire; (augmenté de) Foucault révolutionne l'histoire, Paris, Seuil, 1978, p. 423-425. Paul Veyne rapporte que pour remonter à la source d'un événement historique, il faut toujours le "mettre en intrigue», c'est-à-dire encore d'en faire un "roman vrai». À cette fin, il faut reconstituer la transparence à partir de plusieurs itinéraires politiques, économiques, juridiques, sociaux, etc. 
Jean-François Kerléo. - Il y a deux manières de comprendre cette question. Quels sont les domaines dans lesquels le secret subsiste comme principe et quels sont les secrets persistants là où la transparence s'impose pourtant?

Il n'existe évidemment aucun régime juridique prônant une transparence absolue. Partout où la transparence s'applique, comme dans l'accès au droit, la motivation, la publicité de l'audience et bien d'autres, des exceptions protègent le secret qui apparaît, dans certaines circonstances, comme mieux à même de garantir les principes que l'on cherche à protéger par la transparence elle-même. Comme l'affirme la CEDH par exemple, les droits du justiciable sont protégés par la publicité des débats mais, dans des procès impliquant des mineurs par exemple, le huis clos remplit mieux cette même fonction. La dialectique entre transparence et secret est en mouvement perpétuel, car elle doit être ajustée à chaque nouvelle situation ${ }^{19}$.

Le régime des exceptions à la transparence mérite que l'on s'y attarde, tant leur qualification est parfois difficile à opérer. Par exemple, la CADA est-elle chargée en vertu de la loi du 17 juillet 1978 de distinguer entre les documents préparatoires, inachevés et secrets, ce qui n'est pas une mince affaire? Chacune des exceptions vaut d'ailleurs la peine d'être étudiée: pourquoi les documents inachevés ne sont-ils pas communicables et pourquoi ceux préparatoires ne le sont-ils qu'une fois la décision finale prise? En somme, la transparence n'est admise que si elle n'interfère pas dans l'action politique, ce qui signifie que l'opinion des électeurs n'est éclairée qu'une fois la décision prise. En somme, la transparence vient justifier une décision et non nourrir le débat. L'efficacité de l'action publique est d'ailleurs souvent opposée à la transparence, laquelle serait trop contraignante et restreindrait abusivement la marge de manœuvre des pouvoirs publics. On retrouve ici le problème de la restriction de la liberté d'expression, qui n'est légitime que si elle n'empêche pas le pouvoir de décider seul.

Il n'y a rien d'original à affirmer que le secret est la règle en matière de défense nationale, de sécurité publique et de diplomatie. Même si la

19. On constate actuellement une certaine inversion du raisonnement juridique visà-vis de la transparence. Celle-ci, notamment dans sa déclinaison administrative, s'attaque à l'origine au secret de la puissance publique, la constituant en exception du droit commun de l'administration. Or, apparaissent désormais de nouvelles règles juridiques ayant pour objet non plus de s'attaquer au secret, mais de limiter la transparence elle-même, ce qui aboutit à un schéma original : le secret limité par une transparence, entendue comme série d'exceptions, elle-même dorénavant limitée par de nouveaux secrets, constitutifs juridiquement d'exception à l'exception, etc. 
question de la transparence s'est récemment posée lors du vote de la loi sur le renseignement, à propos du contentieux de la mise en œuvre des techniques de renseignement soumises à autorisation et des fichiers intéressant la sûreté de l'État, c'est à une formation spécialisée ou restreinte du Conseil d'État qu'a été confiée cette compétence ${ }^{20}$. Il en ressort une très grande opacité du régime qui n'est pas vraiment surprenante. En période de circonstances exceptionnelles, en l'occurrence sous le régime de l'état d'urgence, la transparence se trouve réduite, surtout en ce qui concerne les activités de police administrative et judiciaire. C'est ici que des autorités telles que le défenseur des droits, qui a dénoncé les perquisitions abusives effectuées sous le régime de l'état d'urgence, jouent un rôle de premier plan en ce qu'elles peuvent, grâce à la publicité de leurs rapports, interpeller la société civile.

C'est sans doute un secret trop pesant, mais aussi indispensable dans une certaine mesure, qui a conduit à faire émerger une transparence spontanée et non officielle en matière diplomatique. L'affaire Wikileaks et la violence des réactions qu'elle a suscitées de la part des pouvoirs publics sont symptomatiques du secret qui règne dans ce domaine. Or, sans une part de transparence, il est impossible de départager la raison d'État du mensonge. Là où règne le secret, on doit se fier à la parole publique, laquelle n'a pas toujours montré une grande fiabilité dans la justification de l'engagement des forces armées sur le terrain. Aborder les relations internationales sous l'angle de la transparence fait apparaître des questions tout à fait originales sur la place d'une opinion publique internationale et l'apparition d'une distinction entre intérêt général national et mondial, sur l'ambiguïté de la représentation nationale dans une société mondialisée, etc.

Dans une perspective de recherche universitaire, ces différents domaines que sont la diplomatie et la sécurité mériteraient une analyse aussi bien historique que comparative. Les États réagissent différemment aux révélations, à l'activité des médias et des lanceurs d'alerte, et l'histoire politique et internationale façonne des conceptions diplomatiques et sécuritaires de la transparence qui n'ont pas encore été étudiées.

Aymeric Potteau. - Quels sont les apports de vos recherches et de la recherche en général sur la question au cours des dernières années? Que reste-t-il à écrire sur la transparence en matière administrative, constitutionnelle, financière?

20. L. $\mathrm{n}^{\mathrm{o}}$ 2015-912, 24 juil. 2015 relative au renseignement, JO 26 juil. 2015, p. 7-29. 
Jean-François Kerléo. - Évidemment, je ne suis pas le mieux placé pour apprécier l'apport de mes travaux, d'autant plus que, comme je l'évoquais précédemment, l'influence des universitaires sur les pouvoirs publics et le contenu des réformes reste limitée. Dans ma thèse, qui a été soutenue avant l'adoption des lois du 11 octobre 2013, j'avais anticipé l'apparition en France d'une transparence personnalisée par le biais des déclarations de patrimoine et d'intérêts. En ce sens, les travaux universitaires comportent une part de prospective qui prévient certaines dérives ou met en garde sur les dangers de certaines déclinaisons de la transparence.

Je rappelle également que la transparence n'est pas une fin en soi mais un moyen pour parvenir à cette fin. Trop souvent, la transparence est mobilisée comme un objectif qui se confond avec les buts, notamment de démocratisation de l'État, d'assainissement de la vie publique, de régulation de l'économie, d'égalité des candidats à une élection. Ces fins deviennent superflues au regard de la revendication de transparence, suffisante en elle-même, ce qui appauvrit considérablement le débat. C'est pourquoi le chercheur en droit ne peut se contenter d'étudier une réforme, un texte, une jurisprudence, sans se soucier du sens de cette notion à l'intérieur du système politique. Étudier la transparence, c'est réfléchir à sa capacité d'atteindre, sans trop de dommages collatéraux, les objectifs qui la sous-tendent.

De nombreuses perspectives de recherche sont envisageables et plusieurs thèses ou ouvrages sont en préparation à Lyon sur la déontologie publique, intégrant des problématiques de transparence. Le quinquennat de François Hollande aura été marqué par une succession de textes sur la déontologie (élus et collaborateurs, magistrats, fonctionnaires, lobbies, lanceur d'alerte, etc.) qui apportent de nouveaux éléments de réflexion sur la transparence. Jusqu'où la transparence exigée des magistrats est-elle possible sans déstabiliser le procès et risquer des demandes constantes de récusation de la part des justiciables ou des procédures excessives de déport?

À mon sens, il manque des travaux empiriques qui permettraient d'apprécier l'attitude de ceux à qui est imposée la transparence. Il serait intéressant d'étudier l'application de la déontologie au sein des services administratifs (ou d'une juridiction) sous l'angle de la confrontation entre la transparence (déclaration de patrimoine et d'intérêts, entretien déontologique, lanceur d'alerte, information du public, etc.) et les principes de la fonction publique (ou de la magistrature) tels que la neutralité, l'obligation de réserve, de secret professionnel, de discrétion, d'obéissance hiérarchique, etc. Une étude de terrain auprès du référent 
déontologue des centres de gestion ou de la commission de déontologie de la fonction publique, qui voit ses attributions élargies, apporterait incontestablement de nouvelles perspectives à la recherche. L'approche empirique permettrait d'établir les services qui connaissent le plus de difficultés et de faire des propositions concrètes pour combler les lacunes des dispositifs existants.

Le rapport entre la transparence et la participation ouvre également de belles promesses de recherche, notamment avec la combinaison du développement de l'entendement citoyen par la politique d'ouverture des données publiques et les nouvelles procédures de participation telles que les consultations sur Internet, les amendements citoyens (voir la loi sur la République numérique) et la réapparition des pétitions citoyennes. Une dimension empirique serait ici aussi bénéfique pour la recherche dans la mesure où le travail des élus sur leur circonscription révèle parfois l'existence de pratiques de transparence et de participation très innovantes. Par exemple, la mise en œuvre par certains parlementaires de jury citoyen pour l'usage de la réserve parlementaire mériterait qu'on s'y attarde afin d'étudier les modalités de sélection des personnes concernées et le type de procédures retenu pour évaluer les projets subventionnés.

Enfin, concernant spécifiquement la transparence, il manque à l'heure actuelle, mais je m'attelle à combler ce vide, une perspective comparatiste. La transparence s'est universalisée dans la diversité, tant l'imagination du législateur, et parfois du constituant, s'avère débordante en matière de communication et d'accès au droit. Les juristes français se sont intéressés assez tard au droit comparé, ceci s'expliquant par le fait que le droit français a longtemps été une référence à l'étranger et que l'organisation de l'État s'est exportée dans le monde (et continue son exportation comme l'atteste le modèle français des cabinets ministériels qui connaît un certain succès). En matière de transparence administrative, la France a d'ailleurs constitué un modèle pour l'Union européenne et ses États membres puisqu'elle fut incontestablement un précurseur dans les années 1970 en ouvrant l'accès aux documents administratifs et en protégeant les données personnelles. Elle reste à l'avant-garde en matière d'ouverture des données publiques même si d'autres États, tels que le Royaume-Uni qui est à la pointe en ce domaine, pourraient constituer des sources d'inspiration légitimes.

Sur les aspects de la transparence qui recoupent la déontologie, la France était plutôt à la traîne vis-à-vis de la plupart des États. La conception du conflit d'intérêts varie fortement d'un État à l'autre, ce qui permet de pénétrer des systèmes où la place de l'administration ou du 
juge est différente, la liberté d'expression plus ou moins limitée par des considérations patrimoniales ou privées. En la matière, le droit comparé est particulièrement instructif tant les régimes juridiques sont variés, notamment quant au contenu des déclarations (les intérêts des parents et enfants des élus sont-ils publiés contrairement à la France, et pourquoi), à la place de l'open data et aux modes de réutilisation des données, aux répercussions sur la lutte contre la corruption, à la participation citoyenne, etc. Il y a là de quoi reconsidérer notre système juridique à la lumière d'autres droits, cultures, idéologies.

Aymeric Potteau. - Êtes-vous optimiste quant à l'avenir des recherches en matière de transparence? Pensez-vous qu'il s'agisse d'un champ d'analyse dont la doctrine va s'emparer?

Jean-François Kerléo. - La transparence, et plus généralement les problématiques de la déontologie publique, connaissent un regain d'intérêt auprès des chercheurs de différentes disciplines. L'équipe de droit public de l'université Jean-Moulin-Lyon 3 en a fait son axe de recherche principal, et la déontologie est abordée dans plusieurs colloques et journées d'études, notamment à Sciences Po, à partir et autour de la doctrine de son père fondateur, Jeremy Bentham ${ }^{21}$. Le dynamisme de la HATVP contribue également à intégrer les problématiques de la transparence à divers travaux, aussi bien de l'administration que de l'université, permettant aux politistes, aux sociologues, et aux journalistes également, de les faire vivre plus activement dans la sphère publique. La nomination, à deux reprises, d'un universitaire au poste de déontologue de l'Assemblée nationale, démontre que les chercheurs sont en prise directe avec la transparence de la vie publique.

Toutefois, cet objet d'étude reste assez marginal auprès des juristes pour plusieurs raisons. Tout d'abord, la transparence n'est peut-être pas aussi attrayante que les grandes notions sur lesquelles les juristes se focalisent, telles que la liberté, l'égalité, la propriété, etc. Ce manque de prestige peut s'expliquer par sa nouveauté qui l'empêche d'accéder au rang de notion fondatrice de l'État, du droit, du marché, etc. C'est un tort dans la mesure où la transparence constitue une préoccupation du droit au moins depuis le $\mathrm{xv}^{\mathrm{e}}$ siècle, même si le terme n'apparaît pas en tant que tel. Cette nouveauté lui attribue, par ailleurs, un aspect «vulgaire». D’une part, la transparence est protéiforme: elle se trouve

21. Guillaume Tusseau (dir.), La déontologie publique: trajectoire et présence d'une notion ambiguë, colloque de l'école de droit de Sciences Po, 14 mai 2014, à paraître. 
employée, souvent comme un raccourci intellectuel, ce qui en fait une notion slogan, peu rigoureuse, dans la plupart des disciplines juridiques avec des définitions et des approches différentes. La doctrine, qui l'utilise intuitivement plus que scientifiquement, abuse de son usage au point de lui donner une apparence plus journalistique que juridique. D'autre part, elle est associée, dans notre imaginaire collectif, à des idées négatives comme la délation ou encore le voyeurisme, et le développement de la déontologie encourage parfois ces jugements.

Ensuite, je l'ai dit précédemment, le juriste se sent désarmé face à la transparence qui semble enrichir davantage le travail du sociologue et de l'économiste. Il importe peu au juriste d'accéder aux documents administratifs comme le permet la loi du 17 juillet 1978 ou bien de connaître le contenu des déclarations de patrimoine, tandis que le sociologue en tirera de nouvelles sources d'informations et élaborera des statistiques. J'ai contesté plus haut cette approche assez répandue qui s'explique par la clôture disciplinaire à l'université. En effet, la transparence ouvre de nouveaux horizons de recherche pour le juriste qui accepte de s'associer à d'autres disciplines. Les travaux des sociologues sur l'origine des élus enrichissent les théories juridiques sur représentation et représentativité, scrutin majoritaire et scrutin proportionnel, etc. L'évaluation en termes de coûts de transaction des données publiques nourrit les réflexions du juriste sur-le-champ de la concurrence, son efficacité pour les deniers publics. Pour être pleinement exploitée, la transparence impose de nouvelles collaborations scientifiques et techniques.

Enfin, parmi de nombreuses autres explications, je retiendrai également le fait que le juriste s'adresse principalement à ses pairs et aux techniciens/praticiens du droit. D'une part, il a limité son public aux avocats, éventuellement aux juges, et à certains techniciens. D’autre part, il se focalise sur la jurisprudence au détriment de l'institutionnel et délaisse la vie du droit extrajuridictionnelle. Or, par exemple, la transparence relève de la vie intérieure de l'administration qui est parfois commentée par la science administrative, discipline carrefour, mais pas par la science du droit stricto sensu. Ainsi la transparence est centralisée par les administrations qui l'enrichissent progressivement, sans qu'un regard extérieur s'exprime et qu'une analyse juridique soit proposée. Le premier ouvrage sur la transparence administrative était rédigé par des 
conseillers d'État ${ }^{22}$, et la «doctrine organique» s'interroge, depuis des années déjà, sur la déontologie des fonctionnaires.

J'ai cherché à démontrer, dans mes travaux, que la transparence n'est ni une problématique récente, voire passagère, ni un droit secondaire ou accessoire. Elle mérite d'entrer au panthéon des grandes notions, car elle appartient, voire est consubstantielle, à une culture politique et juridique qu'elle révèle et métamorphose à la fois. Par son omniprésence dans les textes juridiques, elle constitue désormais un des topoï de l'analyse juridique.

Maître de conférences en droit public à l'université Jean-Moulin Lyon 3

22. Bruno Lasserre, Noëlle Lenoir et Bernard Stirn, La transparence administrative, Paris, PUF, 1987. Toutefois, la publication suivante sur cette question, qui sera l'œuvre de la doctrine en sciences administratives et politiques, interviendra l'année suivante (voir Jacques Chevallier, "Le mythe de la transparence administrative», dans Information et transparence administratives, Paris, PUF, 1988). 